\title{
Impact of Space Debris and Space Reflectors on Ground-Based Astronomy
}

\author{
D. McNally ${ }^{1}$ \\ University of London Observatory, \\ Mill Hill Park, London NW7 2QS, UK
}

\begin{abstract}
Astronomical fields under observation are being increasingly crossed by satellites. Such crossings either leave a "trail" on a photographic or CCD image, or corrupt a photographic observation. Such trailing/corruption may render an observation useless for scientific analysis. There is also the much more serious problem posed by suggestions to put solar reflectors in space for Earth illumination, artistic, celebratory or advertising purposes and, by extension, to longer term suggestions of ways to utilise dark time rather than twilight time. It is only a matter of time until the solar reflector becomes proven technology. The time left for decisive action may be very short.
\end{abstract}

\section{Introduction}

Ground-based astronomy is concerned with the detection of faint cosmic signals. In such an activity one is concerned to maintain adequate signal/noise $(\mathrm{S} / \mathrm{N})$ ratio. Anything which degrades $\mathrm{S} / \mathrm{N}$ is to be minimised. Unfortunately operational satellites, defunct satellites and large debris in orbit can reflect sunlight after it has become sufficiently dark on the ground to begin serious astronomical observation. Satellites still illuminated by the Sun will be detected if they cross a field under observation: if a photographic plate or CCD camera is being used, a bright satellite will leave a trail across the image; if a photometer is being used, the entire observation will be lost. An astronomical image carrying satellite trails may not be entirely useless: degradation will depend on the purpose for which the image was taken. However, it now has unfortunately become accepted that modern Sky survey plates will be trailed.

There have been recurring suggestions for placing reflectors in space to reflect sunlight. While such suggestions have not yet been implemented successfully, the development of solar reflector technology has reached the point of trial. Were such technology to be put into commercial use, e.g. for advertising purposes, the outlook for ground-based astronomy would indeed be bleak.

Space communications have entered the age of laser communication links. ESA uses this technology for the SPOT4 mission. This has brought about a new situation for optical astronomy, akin to that of radio astronomy, where ground-

${ }^{1}$ Current address: 17 Greenfield, Hatfield, Herts AL9 5HW, UK 
based optical observatories will be swept by laser communication beams just as ground-based radio observatories are swept by radio communications. Thus far laser communication is taking place in the far red at a wavelength close to $800 \mathrm{~nm}$ and, in the case of SPOT4, for intersatellite communication.

Another "possible" hazard to optical astronomy may have been revealed (Ohishi 2001): the HAPS radio relay balloon project. This is clearly a project to be evaluated for its possible hazard to urban observatories located near large centres of population.

\section{Measures of Satellite Trails}

Satellite trails do not give good statistical data, since image-taking activity varies from year to year and the distribution of times at which images are taken varies from night to night. However, in any collection of plates the numbers will rapidly dwindle as the selection criteria are increased in number.

If one looks at an entire sample taken over a period of years, then one must accept inhomogeneity of data. Since satellite trailing is most likely within two hours after sunset or two hours before sunrise the sample will be biased by the number of plates taken within these periods. However, a large data set, while inhomogeneous, may be used to look at long-term trends. Tritton and Norton (private communication, 1995) have examined the UK Schmidt Plates for satellite trails for the period $1977 / 93$. However, because of a change of filter, only the data for $1985 / 93$ were selected. The results are set out in Table 1 (McNally and Rast 1999):

Table 1. Trail and Satellite number data

\begin{tabular}{lcccc} 
Year & $\begin{array}{c}\text { Number of trails } \\
\text { per 60 min exposure }\end{array}$ & $\begin{array}{c}\text { Number of Spacecraft } \\
\text { on orbit }\end{array}$ & $I_{T}$ & $I_{S}$ \\
1985 & 2.5 & 1500 & 1.1 & 0.9 \\
$1987^{*}$ & 2.3 & 1624 & 1.0 & 1.0 \\
1989 & 2.7 & 1749 & 1.2 & 1.1 \\
1991 & 2.4 & 1916 & 1.0 & 1.2 \\
1993 & 2.9 & 2084 & 1.3 & 1.3 \\
\hline
\end{tabular}

* Year used to normalize data.

1 Tritton and Norton (1995). ${ }^{2}$ Orbital Debris 1995, p.20, Fig.1.2.

Trail rate is given as the mean annual rate per 60 -min exposure. The number of spacecraft on orbit is also given. Indices $I_{T}$ and $I_{S}$ are normalised values to further illustrate the trend. It is clear from the table that the satellite on orbit population increases steadily at $4 \%$ per annum. The satellite trail rate, while varying erratically for reasons given above, shows an increasing trend at $2 \%$ per annum. McNally (1997) has unsuccessfully sought increases in trail rate associated with major satellite breakups. We may conclude that trailing is related to satellite numbers. In a visual inspection of the Mt. Palomar/ESO B-survey of the northern sky, we found that while there is an average of one trail per plate, $50 \%$ of plates were untrailed, $25 \%$ carried one trail and $25 \%$ carried 
multiple trails (in one case carrying 11 trails). Overall, one in two plates exhibit trails.

Murdin (1991) has pointed out that if those plates in the UK Schmidt collection taken within one to two hours of sunset or sunrise are considered, all plates are trailed, with an average of five trails per plate.

These figures suggest that doubling up satellite numbers will lead to significant increase in trail rates. Plates with 11 trails will soon not be a noteworthy rarity but a common occurrence. As trail rate increases, the chances increase that any image carrying trails will be unsuited for its scientific purpose.

One should also note that certain satellites, e.g. Mir and TiPS, are both bright and extended. TiPS subtends a maximum angle of $7^{\prime}$ on the sky, which is comparable with the field of many commonly used CCD cameras. While TiPS is just on the limit of naked eye visibility, Mir is readily visible to the naked eye. The International Space Station will certainly be both a readily visible and an extended object.

It is not easy to translate such statistics into the outlook for photometry. Clearly photometric fields of $15^{\prime \prime} \times 15^{\prime \prime}$ are very much smaller than the $6^{\circ} \times 6^{\circ}$ deep sky survey fields. On the other hand photometric observations are susceptible to the presence of far fainter satellites, namely those several magnitudes fainter than the object being measured. In a photometric field, satellites of brightness down to the sky limit could be involved for the faintest observations. On the other hand, for a deep sky survey plate, a satellite moving at about $0.5^{\circ} \mathrm{s}^{-1}$, will cross a sky survey plate in less than $17 \mathrm{~s}$. Clearly only the brighter satellites are of significance for such plates.

\section{Other Hazards from Space}

There have been numerous proposals over the years to put highly illuminated objects in space: objects easily visible with the naked eye from the ground, e.g. the Ring of Light, the Star of Tolerance, Znamya 2 and 2.5. Thus far such objects are usually proposed in the form of a solar reflector and therefore confined to twilight hours. Only Znamyas 2 and 2.5 became reality. To date none has been successfully demonstrated, but all pose a severe threat to future astronomy. Were they to become a feature of "civilised" living, for celebratory, artistic or advertising purposes, then the limitations of the solar reflector would become evident and constraining. There has been a proposal already, calling for space holograms for advertising. Holograms would be a feature of the dark sky, not just the near twilight sky as with solar reflectors. Holograms could feature throughout the dark hours. Since the brightness of reflectors is now being assessed in terms of 10-100 times the brightness of the full Moon, one does not suppose the proposed holograms will be significantly fainter! The natural Moon is of sufficient brightness to rule out deep sky astronomy for a full half of every month. Were many man-made "super moons" to become a feature of the night sky then optical astronomy from the ground would be seriously emasculated. This is not thinking unrealistically. Significant optical astronomy would not be possible in the bright sky such reflectors and holograms would create.

Others are looking hard at other uses of solar reflectors, for example to collect solar radiation for conversion to microwaves and then transmission to 
Earth for large-scale power generation. It is already admitted that such programmes are likely to ruinously damage ground-based radio astronomy and give optical and ground-based infrared astronomy very serious problems. The proposals are there now. The need for alternative sources of power generation is not in dispute, but the cost to sustained astronomical research would be the end of ground-based astronomy as it is now known.

\section{Action}

What action can be taken? In the case of operational satellites, defunct satellites and large debris, there is nothing that can be done on the basis of existing technology. Satellites will continue to increase in number on decadic timescales and deorbit only on century or millennial timescales. Trailing of fields under observation will continue to be a worsening problem. It is probably unreasonable to expect that satellites can be made less reflecting. Astronomy at both optical and infrared wavelengths will have to become used to the idea that, in the periods within two hours of sunset or sunrise, trailing will be the norm and that norm will only grow more unfavourable. We can develop techniques which could offset some of the consequences of trailing, but it is too early to predict how comprehensive such techniques should become. Sadly, the price to be paid for rapid accumulation of satellites on orbit will be paid by astronomy through loss of telescope usage.

In the case of bright solar reflectors and their putative progeny, it is not too late to take action, though time is undoubtedly very short before a solar reflector is successfully demonstrated. We need urgent discussion to ensure that such potentially disastrous creations do not emasculate optical and groundbased infrared astronomy. Astronomy, like any science, is critically dependent on high quality $\mathrm{S} / \mathrm{N}$ data. Without a sustained flow of such data, astronomy will surely wither away. Wide agreement on this issue must be sought on an international basis. The need for agreement is very urgent. The next trial of Znamya, Znamya 3, is thought to be planned for 2000 or shortly thereafter.

Astronomy is now confronted with the prospect of space laser communication at optical (far red) wavelengths. Such a communications system has already been implemented by ESA with SPOT4. This is a situation which merits detailed discussion to assess how significant a threat this might be for the future and to examine how close a parallel such laser communication may create with the current situation for radio astronomy.

Finally HAPS (High Altitude Platform Systems), the placing of radio communication relay balloons over major centres of population at a height of $20 \mathrm{~km}$, may have repercussions on optical astronomy, by reflecting city lights and by being an obstruction. Given a balloon dimension of $150 \mathrm{~m}$, an observatory $20 \mathrm{~km}$ from the sub-balloon point would see it as an object with an angular extent of $15^{\prime}$, again comparable with the field of common CCD cameras. Further investigation is urgently needed to assess the impact for HAPS on urban observatories. 


\section{Conclusion}

Trailing of astronomical fields of view is an increasing hazard. While we may live with current levels of trailing, that may not be an option in the future. Trailing will continue to be a hazard for of the order of several hours per location per night, and the increasing loss of efficiency in operating telescopic facilities will become unacceptable. We have to recognise that that loss is imposed by the actions of others. Interference with the legitimate activities of one group by the legitimate actions of others is something the UN expects to resolve. Satellite trailing of astronomical fields is a classic example of such a situation.

There are those who argue that the ultimate solution for astronomy is to transfer all observational work to the far side of the Moon. The exciting possibilities that such a proposal raises should not blind us to the fact that it is not an option for astronomy now, nor in the foreseeable future, and to so argue verges on deception. Since it is only a matter of years until a successful solar reflector will be demonstrated, it is now urgent to examine the consequences for astronomy, particularly as an initial use for such reflectors may be for advertising. Should it be established that advertising from space is acceptable, then the consequences for astronomy at optical and infrared wavelengths could be severe. The limitations of solar reflectors would become all too apparent and advertisers would seek to exploit technology which could utilise the entire night sky. Should the sky become full of bright artificial Moons in perpetuity, the science of astronomy could not compete: even spectroscopic observations could not survive.

Astronomy also must urgently investigate the impact of laser communication. Radio communication has not been a happy precedent and the parallels do not engender confidence. The appearance of HAPS is an indication that new creative schemes will be continually proposed and that astronomical science will have to exercise continued vigilance.

I hope our fears prove groundless. Past experience does not encourage optimism. Yet the future for astronomical science holds such exciting promise. The fact that astronomy continues to survive in spite of growing adverse environmental impact surely means that we should not despair.

\section{References}

McNally, D. 1997, Adv.Space Res., 19, 399-402

McNally, D. and Rast, R.H. 1999, Adv.Space Res., 23, 255-258

Murdin, P. 1991, In IAU Colloquium 112, Light Pollution, Radio Interference and Space Debris, Ed. D. L. Crawford, Astr. Soc. Pacific Conf. Ser., Vol. 17, p.141

Ohishi, M. 2001, in these proceedings

Orbital Debris - a Technical Assessment, 1995, National Research Council, National Academy Press.

Tritton, S. and Norton, L. 1995, private communication. 